\title{
Oscillation of certain higher-order neutral partial functional differential equations
}

\author{
Wei Nian Li* and Weihong Sheng
}

*Correspondence: wnli@263.net

Department of Mathematics, Binzhou University, Binzhou, Shandong 256603, People's

Republic of China

\section{Abstract}

In this paper, we study the oscillation of certain higher-order neutral partial functional differential equations with the Robin boundary conditions. Some oscillation criteria are established. Two examples are given to illustrate the main results in the end of this paper.

Keywords: Oscillation, Partial functional differential equation, Robin boundary condition

Mathematics Subject Classification: 35B05, 35R10

\section{Background}

It is well known that the theory of partial functional differential equations can be applied to many fields, such as population dynamics, cellular biology, meteorology, viscoelasticity, engineering, control theory, physics and chemistry (Wu 1996). In the monograph, Wu (1996) provided some fundamental theories and applications of partial functional differential equations.

The oscillation theory as a part of the qualitative theory of partial functional differential equations has been developed in the past few years. Many researchers have established some oscillation results for partial functional differential equations. For example, see the monograph (Yoshida 2008) and the papers (Bainov et al. 1996; Fu and Zhuang 1995; Li and Cui 1999; Li 2000; Li and Cui 2001; Ouyang et al. 2005; Gao and Luo 2008; $\mathrm{Li}$ and Han 2006; Wang et al. 2010). We especially note that the monograph (Yoshida 2008) contained large material on oscillation theory for partial differential equations.

$\mathrm{Li}$ and Cui (2001) studied the oscillation of even order partial functional differential equations

$$
\begin{aligned}
& \frac{\partial^{n}}{\partial t^{n}}[u(x, t)+\mu(t) u(x, t-\rho)]=a(t) \Delta u(x, t)+\sum_{k=1}^{s} a_{k}(t) \Delta u\left(x, \rho_{k}(t)\right) \\
& \quad-q(x, t) u(x, t)-\int_{a}^{b} p(x, t, \xi) u(x, g(t, \xi)) \mathrm{d} \sigma(\xi), \quad(x, t) \in \Omega \times[0, \infty) \equiv G,
\end{aligned}
$$

where $n \geq 2$ is an even integer, with the two kinds of boundary conditions:

(c) $2016 \mathrm{Li}$ and Sheng. This article is distributed under the terms of the Creative Commons Attribution 4.0 International License (http://creativecommons.org/licenses/by/4.0/), which permits unrestricted use, distribution, and reproduction in any medium, provided you give appropriate credit to the original author(s) and the source, provide a link to the Creative Commons license, and indicate if changes were made. 


$$
\frac{\partial u(x, t)}{\partial N}+v(x, t) u(x, t)=0, \quad(x, t) \in \partial \Omega \times[0, \infty)
$$

and

$$
u(x, t)=0, \quad(x, t) \in \partial \Omega \times[0, \infty) .
$$

Ouyang et al. (2005) established the oscillation of odd order partial functional differential equations

$$
\begin{gathered}
\frac{\partial^{n} u(x, t)}{\partial t^{n}}-a(t) \Delta u(x, t)-\sum_{k=1}^{s} p_{k}(x, t) u\left(x, t-\sigma_{k}\right)-\sum_{j=1}^{m} q_{j}(x, t) u\left(x, t-\tau_{j}\right) \\
+h(t) f\left(u\left(x, t-r_{1}\right), \cdots, u\left(x, t-r_{\ell}\right)\right)=0, \quad(x, t) \in \Omega \times[0, \infty) \equiv G,
\end{gathered}
$$

where $n$ is an odd integer and $s \leq m$, with the boundary conditions $(B 1),(B 2)$ and

$$
\frac{\partial u(x, t)}{\partial N}=0, \quad(x, t) \in \partial \Omega \times[0, \infty) .
$$

In this paper, we investigate the oscillation of the following higher-order neutral partial functional differential equations

$$
\begin{gathered}
\frac{\partial^{n}}{\partial t^{n}}[u(x, t)+\mu(t) u(x, t-\tau)]=a(t) \Delta u(x, t)+\sum_{k=1}^{s} a_{k}(t) \Delta u\left(x, \rho_{k}(t)\right) \\
-\int_{a}^{b} p(t, \xi) u(x, g(t, \xi)) \mathrm{d} \sigma(\xi), \quad(x, t) \in \Omega \times[0, \infty) \equiv G,
\end{gathered}
$$

with the Robin boundary condition

$$
\alpha(x) \frac{\partial u(x, t)}{\partial N}+\beta(x) u(x, t)=0, \quad(x, t) \in \partial \Omega \times[0, \infty),
$$

where $n \geq 2$ is an even integer, $\Omega$ is a bounded domain in $\mathbb{R}^{M}$ with a piecewise smooth boundary $\partial \Omega$, and $\Delta$ is the Laplacian in the Euclidean $M$-space $\mathbb{R}^{M}, \alpha, \beta \in C(\partial \Omega,[0, \infty)$, $\alpha^{2}(x)+\beta^{2}(x) \neq 0$, and $N$ is the unite exterior normal vector to $\partial \Omega$.

Throughout this paper, we always suppose that the following conditions hold:

(C1) $\mu \in C^{n}([0, \infty) ;[0, \infty)), 0 \leq \mu(t) \leq 1, \tau=$ const. $>0$;

(C2) $a, a_{k} \in C([0, \infty) ;[0, \infty)), \rho_{k} \in C([0, \infty) ;[0, \infty)), \rho_{k}(t) \leq t$, $\lim _{t \rightarrow+\infty} \rho_{k}(t)=+\infty, k \in I_{s}=\{1,2, \ldots, s\}$;

(C3) $p \in C([0, \infty) \times[a, b] ;[0, \infty)), \quad g \in C([0, \infty) \times[a, b] ;[0, \infty)), \quad g(t, \xi) \leq t$, $\xi \in[a, b], g(t, \xi)$ is a nondecreasing function with respect to $t$ and $\xi$, respectively, and $\lim _{t \rightarrow+\infty} \inf _{\xi \in[a, b]}\{g(t, \xi)\}=+\infty$;

(C4) $\sigma \in([a, b] ; \mathbb{R})$ and $\sigma(\xi)$ is nondecreasing in $\xi$, the integral in (1) is Stieltjes integral. 
As it is customary, the solution $u(x, t) \in C^{n}(G) \bigcap C^{1}(\bar{G})$ of the problem (1), (2) is said to be oscillatory in the domain $G \equiv \Omega \times[0, \infty)$ if for any positive number $\mu$ there exists a point $\left(x_{0}, t_{0}\right) \in \Omega \times[\mu, \infty)$ such that the equality $u\left(x_{0}, t_{0}\right)=0$ holds.

To the best of our knowledge, no result is known regarding the oscillatory behavior of higher-order partial functional differential equations with the Robin boundary condition (2) up to now.

The paper is organized as follows. In "Main results" section, we establish some results for the oscillation of the problem (1), (2). In "Examples" section, we construct two examples to illustrate our main results.

\section{Main results}

In this section, we establish the oscillation criteria of the problem (1), (2). First, we introduce the following lemma which is very useful for establishing our main results.

Lemma 1 Ye and Li (1990). Suppose that $\lambda_{0}$ is the smallest eigenvalue of the problem

$$
\left\{\begin{array}{l}
\Delta \varphi(x)+\lambda \varphi(x)=0, \quad \text { in } \Omega, \\
\alpha(x) \frac{\partial \varphi(x)}{\partial N}+\beta(x) \varphi(x)=0, \quad \text { on } \partial \Omega
\end{array}\right.
$$

and $\varphi(x)$ is the corresponding eigenfunction of $\lambda_{0}$. Then $\lambda_{0}=0, \varphi(x)=1$ as $\beta(x)=0$ $(x \in \Omega)$ and $\lambda_{0}>0, \varphi(x)>0(x \in \Omega)$ as $\beta(x) \not \equiv 0(x \in \partial \Omega)$.

Next, we give our main results.

Theorem 2 If $\beta(x) \not \equiv 0$ for $x \in \partial \Omega$, then the necessary and sufficient condition for all solutions of the problem (1), (2) to oscillate is that all solutions of the differential equation

$$
\begin{gathered}
{[y(t)+\mu(t) y(t-\tau)]^{(n)}+\lambda_{0} a(t) y(t)+\lambda_{0} \sum_{k=1}^{s} a_{k}(t) y\left(\rho_{k}(t)\right)} \\
\quad+\int_{a}^{b} p(t, \xi) y(g(t, \xi)) \mathrm{d} \sigma(\xi)=0, \quad t \geq 0,
\end{gathered}
$$

to oscillate, where $\lambda_{0}$ is the smallest eigenvalue of (3).

Proof (i) Sufficiency. Suppose to the contrary that there is a non-oscillatory solution $u(x, t)$ of the problem (1), (2) which has no zero in $\Omega \times\left[t_{0}, \infty\right)$ for some $t_{0} \geq 0$. Without loss of generality we assume that $u(x, t)>0, u(x, t-\tau)>0, u\left(x, \rho_{k}(t)\right)>0$, $u(x, g(t, \xi))>0,(x, t) \in \Omega \times\left[t_{1}, \infty\right), k \in I_{s}$.

Multiplying both sides of (1) by $\varphi(x)$ and integrating with respect to $x$ over the domain $\Omega$, we have

$$
\begin{aligned}
\frac{\mathrm{d}^{n}}{\mathrm{~d} t^{n}}\left[\int_{\Omega} u(x, t) \varphi(x) \mathrm{d} x+\mu(t) \int_{\Omega} u(x, t-\tau) \varphi(x) \mathrm{d} x\right] \\
=a(t) \int_{\Omega} \Delta u(x, t) \varphi(x) \mathrm{d} x+\sum_{k=1}^{s} a_{k}(t) \int_{\Omega} \Delta u\left(x, \rho_{k}(t)\right) \varphi(x) \mathrm{d} x \\
\quad-\int_{\Omega} \int_{a}^{b} p(t, \xi) u(x, g(t, \xi)) \varphi(x) \mathrm{d} \sigma(\xi) \mathrm{d} x, \quad t \geq t_{1} .
\end{aligned}
$$


From Green's formula and boundary condition (2), it follows that

$$
\begin{aligned}
\int_{\Omega} & \Delta u(x, t) \varphi(x) \mathrm{d} x \\
= & \int_{\partial \Omega}\left(\varphi(x) \frac{\partial u(x, t)}{\partial N}-u(x, t) \frac{\partial \varphi(x)}{\partial N}\right) \mathrm{d} S+\int_{\Omega} u(x, t) \Delta \varphi(x) \mathrm{d} x \\
= & \int_{\partial \Omega}\left(\varphi(x) \frac{\partial u(x, t)}{\partial N}-u(x, t) \frac{\partial \varphi(x)}{\partial N}\right) \mathrm{d} S-\lambda_{0} \int_{\Omega} u(x, t) \varphi(x) \mathrm{d} x, \quad t \geq t_{1},
\end{aligned}
$$

where $\mathrm{d} S$ is the surface element on $\partial \Omega$.

If $\alpha(x) \equiv 0, x \in \partial \Omega$, then from (2) we have

$$
\beta(x) \not \equiv 0, u(x, t)=0, \quad(x, t) \in \partial \Omega \times[0, \infty) .
$$

Hence, we obtain

$$
\int_{\partial \Omega}\left(\varphi(x) \frac{\partial u(x, t)}{\partial N}-u(x, t) \frac{\partial \varphi(x)}{\partial N}\right) \mathrm{d} S \equiv 0, \quad t \geq t_{1} .
$$

If $\alpha(x) \not \equiv 0, x \in \partial \Omega$. Noting that $\partial \Omega$ is piecewise smooth, $\alpha, \beta \in C(\partial \Omega,[0, \infty))$, $\alpha^{2}(x)+\beta^{2}(x) \neq 0$, without loss of generality, we can assume that $\alpha(x)>0, x \in \partial \Omega$. Then by (2) and (3) we have

$$
\begin{aligned}
\int_{\partial \Omega} & \left(\varphi(x) \frac{\partial u(x, t)}{\partial N}-u(x, t) \frac{\partial \varphi(x)}{\partial N}\right) \mathrm{d} S \\
= & \int_{\partial \Omega}\left(-\varphi(x) \frac{\beta(x)}{\alpha(x)} u(x, t)+u(x, t) \frac{\beta(x)}{\alpha(x)} \varphi(x)\right) \mathrm{d} S=0, \quad t \geq t_{1},
\end{aligned}
$$

Therefore, using Lemma 1, we obtain

$$
\int_{\Omega} \Delta u(x, t) \varphi(x) \mathrm{d} x=-\lambda_{0} \int_{\Omega} u(x, t) \varphi(x) \mathrm{d} x, \quad t \geq t_{1} .
$$

Similarly, we have

$$
\int_{\Omega} \Delta u\left(x, \rho_{k}(t)\right) \varphi(x) \mathrm{d} x=-\lambda_{0} \int_{\Omega} u\left(x, \rho_{k}(t)\right) \varphi(x) \mathrm{d} x, \quad t \geq t_{1}, k \in I_{s} .
$$

It is easy to see that

$$
\begin{aligned}
& \int_{\Omega} \int_{a}^{b} p(t, \xi) u(x, g(t, \xi)) \varphi(x) \mathrm{d} \sigma(\xi) \mathrm{d} x \\
& \quad=\int_{a}^{b} p(t, \xi) \int_{\Omega} u(x, g(t, \xi)) \varphi(x) \mathrm{d} x \mathrm{~d} \sigma(\xi), \quad t \geq t_{1} .
\end{aligned}
$$

Set

$$
V(t)=\int_{\Omega} u(x, t) \varphi(x) \mathrm{d} x, \quad t \geq t_{1} .
$$


Combining (5)-(8) we have

$$
\begin{aligned}
& {[V(t)+\mu(t) V(t-\tau)]^{(n)}+\lambda_{0} a(t) V(t)+\lambda_{0} \sum_{k=1}^{s} a_{k}(t) V\left(\rho_{k}(t)\right)} \\
& \quad+\int_{a}^{b} p(t, \xi) V(g(t, \xi)) \mathrm{d} \sigma(\xi)=0, \quad t \geq t_{1} .
\end{aligned}
$$

Obviously, it follows from (9) that $V(t)$ is a positive solution of Eq. (4), which contradicts the fact that all solutions of Eq. (4) are oscillatory.

(ii) Necessity. Suppose that Eq. (4) has a non-oscillatory solution $\widetilde{V}(t)>0$. Without loss of generality we assume $\widetilde{V}(t)>0$ for $t \geq t_{*} \geq 0$, where $t_{*}$ is some large number. From (4), we have

$$
\begin{aligned}
& {[\widetilde{V}(t)+\mu(t) \widetilde{V}(t-\tau)]^{(n)}+\lambda_{0} a(t) \widetilde{V}(t)+\lambda_{0} \sum_{k=1}^{s} a_{k}(t) \widetilde{V}\left(\rho_{k}(t)\right)} \\
& \quad+\int_{a}^{b} p(t, \xi) \widetilde{V}(g(t, \xi)) \mathrm{d} \sigma(\xi)=0, \quad t \geq t_{*} .
\end{aligned}
$$

Multiplying both sides of (10) by $\varphi(x)$, we obtain

$$
\begin{aligned}
& \frac{\partial^{n}}{\partial t^{n}}[\widetilde{V}(t) \varphi(x)+\mu(t) \widetilde{V}(t-\tau) \varphi(x)] \\
& \quad+\lambda_{0} a(t) \widetilde{V}(t) \varphi(x)+\lambda_{0} \sum_{k=1}^{s} a_{k}(t) \widetilde{V}\left(\rho_{k}(t)\right) \varphi(x) \\
& \quad+\int_{a}^{b} p(t, \xi) \widetilde{V}(g(t, \xi)) \varphi(x) \mathrm{d} \sigma(\xi)=0, \quad t \geq t_{*}, \quad x \in \Omega .
\end{aligned}
$$

Let $\widetilde{u}(x, t)=\widetilde{V}(t) \varphi(x), \quad(x, t) \in \Omega \times[0, \infty)$. By Lemma 1 , we have $\Delta \varphi(x)=$ $-\lambda_{0} \varphi(x), \quad x \in \Omega$. Then (11) implies

$$
\begin{aligned}
& \frac{\partial^{n}}{\partial t^{n}}[\widetilde{u}(x, t)+\mu(t) \widetilde{u}(x, t-\tau)]=a(t) \Delta \widetilde{u}(x, t)+\sum_{k=1}^{s} a_{k}(t) \Delta \widetilde{u}\left(x, \rho_{k}(t)\right) \\
& \quad-\int_{a}^{b} p(t, \xi) \widetilde{u}(x, g(t, \xi)) \mathrm{d} \sigma(\xi), \quad t \geq t_{*}, x \in \Omega
\end{aligned}
$$

which shows that $\widetilde{u}(x, t)=\widetilde{V}(t) \varphi(x),(x, t) \in \Omega \times\left[t_{*}, \infty\right)$, satisfies (1).

From Lemma 1, we get

$$
\alpha(x) \frac{\partial \varphi(x)}{\partial N}+\beta(x) \varphi(x)=0, \quad x \in \partial \Omega,
$$

which implies

$$
\alpha(x) \frac{\partial \widetilde{u}(x, t)}{\partial N}+\beta(x) \widetilde{u}(x, t)=0, \quad(x, t) \in \partial \Omega \times[0, \infty) .
$$

Hence $\widetilde{u}(x, t)=\widetilde{V}(t) \varphi(x)>0$ is a non-oscillatory solution of the problem (1), (2), which is a contradiction. The proof is complete. 
Remark 3 Theorem 2 shows that the oscillation of problem (1), (2) is equivalent to the oscillation of the differential equation (4).

Theorem 4 If $\beta(x) \not \equiv 0$ for $x \in \partial \Omega$, and the neutral differential inequality

$$
[y(t)+\mu(t) y(t-\tau)]^{(n)}+\int_{a}^{b} p(t, \xi) y(g(t, \xi)) \mathrm{d} \sigma(\xi) \leq 0, \quad t \geq 0,
$$

has no eventually positive solutions, then every solution of the problem (1), (2) is oscillatory in $G$.

Proof Suppose to the contrary that there is a non-oscillatory solution $u(x, t)$ of the problem (1), (2) which has no zero in $\Omega \times\left[t_{0}, \infty\right)$ for some $t_{0} \geq 0$. Without loss of generality we assume that $u(x, t)>0, u(x, t-\tau)>0, u\left(x, \rho_{k}(t)\right)>0, u(x, g(t, \xi))>0$, $(x, t) \in \Omega \times\left[t_{1}, \infty\right), k \in I_{s}$. As in the proof of Theorem 2, we obtain Eq. (9). By Lemma 1, from (9) we have

$$
\begin{aligned}
& {[V(t)+\mu(t) V(t-\tau)]^{(n)}+\int_{a}^{b} p(t, \xi) V(g(t, \xi)) \mathrm{d} \sigma(\xi)} \\
& \quad=-\lambda_{0} a(t) V(t)-\lambda_{0} \sum_{k=1}^{s} a_{k}(t) V\left(\rho_{k}(t)\right) \\
& \quad \leq 0, \quad t \geq t_{1},
\end{aligned}
$$

which shows that $V(t)>0$ is a solution of the inequality (14). This is a contradiction. The proof of Theorem 4 is complete.

Using Theorems 1 and 2 in Li and Cui (2001), we can obtain the following two conclusions, respectively.

Theorem 5 Assume that $\beta(x) \not \equiv 0$ for $x \in \partial \Omega$. Iffor $t_{0}>0$,

$$
\int_{t_{0}}^{+\infty} \int_{a}^{b} p(s, \xi)[1-\mu(g(s, \xi))] \mathrm{d} \sigma(\xi) \mathrm{d} s=+\infty,
$$

then every solution of the problem (1), (2) is oscillatory in G.

Theorem 6 Assume that $\beta(x) \not \equiv 0$ for $x \in \partial \Omega, \mu(t) \equiv \mu$ is a positive constant, $p(t, \xi)$ is periodic in $t$ with period $\rho$. If for $t_{0}>0$,

$$
\begin{aligned}
& g(t-c, \xi)=g(t, \xi)-c \quad \text { for any number } c>0, \\
& \int_{t_{0}}^{+\infty} \int_{a}^{b} p(s, \xi) \mathrm{d} \sigma(\xi) \mathrm{d} s=+\infty
\end{aligned}
$$

then every solution of the problem (1), (2) is oscillatory in G. 


\section{Examples}

In this section, we give two examples to illustrate our main results.

Example 7 Consider the partial functional differential equation

$$
\begin{gathered}
\frac{\partial^{6}}{\partial t^{6}}\left[u(x, t)+\frac{1}{5} u(x, t-\pi)\right]=3 \Delta u(x, t)+\frac{11}{5} \Delta u\left(x, t-\frac{3 \pi}{2}\right) \\
-\int_{-\pi}^{-\pi / 2} \frac{11}{5} u(x, t+\xi) \mathrm{d} \xi, \quad(x, t) \in(0, \pi) \times[0, \infty),
\end{gathered}
$$

with the boundary condition

$$
u(0, t)=u(\pi, t)=0, \quad t \geq 0 .
$$

Here $n=6, \mu(t)=\frac{1}{5}, \tau=\pi, a(t)=3, a_{1}(t)=\frac{11}{5}, \rho_{1}(t)=t-\frac{3 \pi}{2}, p(t, \xi)=\frac{11}{5}, g(t, \xi)$ $=t+\xi, \sigma(\xi)=\xi, a=-\pi, b=-\frac{\pi}{2}$. It is easy to see that for $t_{0}>0$,

$$
\int_{t_{0}}^{+\infty} \int_{a}^{b} p(s, \xi)[1-\mu(g(s, \xi))] \mathrm{d} \sigma(\xi) \mathrm{d} s=\int_{t_{0}}^{+\infty} \int_{-\pi}^{-\pi / 2} \frac{11}{5}\left[1-\frac{1}{5}\right] \mathrm{d} \xi \mathrm{d} s=+\infty .
$$

Then the conditions of Theorem 5 are fulfilled. Therefore every solution of the problem (19), (20) is oscillatory in $(0, \pi) \times[0, \infty)$. Indeed, $u(x, t)=\sin x \cos t$ is such a solution.

Example 8 Consider the partial functional differential equation

$$
\begin{gathered}
\frac{\partial^{4}}{\partial t^{4}}\left[u(x, t)+\frac{1}{2} u(x, t-\pi)\right]=\frac{1}{3} \Delta u(x, t)+\frac{1}{6} \Delta u\left(x, t-\frac{\pi}{2}\right) \\
-\int_{-\pi}^{-\pi / 2} \frac{1}{6} u(x, t+\xi) \mathrm{d} \xi, \quad(x, t) \in(0, \pi) \times[0, \infty),
\end{gathered}
$$

with the boundary condition

$$
u_{x}(0, t)+u(0, t)=u_{x}(\pi, t)+u(\pi, t)=0, \quad t \geq 0 .
$$

Here $n=4, \mu(t)=\frac{1}{2}, \tau=\pi, a(t)=\frac{1}{3}, a_{1}(t)=\frac{1}{6}, \rho_{1}(t)=t-\frac{\pi}{2}, p(t, \xi)=\frac{1}{6}, g(t, \xi)=$ $t+\xi, \sigma(\xi)=\xi, a=-\pi, b=-\frac{\pi}{2}$. It is easy to see that for $t_{0}>0$,

$$
\int_{t_{0}}^{+\infty} \int_{a}^{b} p(s, \xi)[1-\mu(g(s, \xi))] \mathrm{d} \sigma(\xi) \mathrm{d} s=\int_{t_{0}}^{+\infty} \int_{-\pi}^{-\pi / 2} \frac{1}{6}\left[1-\frac{1}{2}\right] \mathrm{d} \xi \mathrm{d} s=+\infty
$$

which shows that the conditions of Theorem 5 are satisfied. By Theorem 5 , we obtain that every solution of the problem (21), (22) is oscillatory in $(0, \pi) \times[0, \infty)$. In fact, $u(x, t)=e^{-x} \cos t$ is such a solution.

\section{Conclusions}

This paper provides some oscillation criteria for solutions of higher-order neutral partial functional differential equations with Robin boundary conditions. Using Lemma 1, we obtain Theorems 2 and 4. We should note that Theorem 2 shows that the oscillation of 
the problem (1), (2) is equivalent to the oscillation of the functional differential equation (4). Using the results in Li and Cui (2001), two useful conclusions are established in Theorems 5 and 6.

Authors' contributions

Both authors contributed equally to the writing of this paper. Both authors read and approved the final manuscript.

Acknowledgements

The authors are grateful to anonymous referees for their kind comments and suggestions on this paper. This work is supported by the National Natural Science Foundation of China (10971018).

\section{Competing interests}

Both authors declare that they have no competing interests.

Received: 31 January 2016 Accepted: 5 April 2016

Published online: 14 April 2016

\section{References}

Bainov D, Cui BT, Minchev E (1996) Forced oscillation of solutions of certain hyperbolic equations of neutral type. J Comput Appl Math 72:309-318

Fu X, Zhuang W (1995) Oscillation of certain neutral delay parabolic equations. J Math Anal Appl 191:473-489

Gao Z, Luo L (2008) Oscillation of solutions of nonlinear neutral hyperbolic partial differential equations with continuous deviating arguments and damped terms. Math Appl 21:399-403 (in Chinese)

LiWN (2000) Oscillation properties for systems of hyperbolic differential equations of neutral type. J Math Anal Appl 248:369-384

LiWN, Cui BT (1999) Oscillation of solutions of neutral partial functional differential equations. J Math Anal Appl 234:123-146

Li WN, Cui BT (2001) A class of even order neutral differential inequalities and its applications. Appl Math Comput 122:95-106

Li WN, Han M (2006) Oscillation tests for certain systems of parabolic differential equations with neutral type. Rocky Mt J Math 36:1285-1300

Ouyang Z, Zhou S, Yin F (2005) Oscillation for a class of odd-order delay parabolic differential equations. J Comput Appl Math 175:305-319

Wang CY, Wang S, Yan X, Li L (2010) Oscillation of a class of partial functional population model. J Math Anal Appl 368:32-42

Wu J (1996) Theory and applications of partial functional differential equations. Springer, New York

Ye QX, Li ZY (1990) Theory of reaction diffusion equations. Science Press, Beijing (in Chinese)

Yoshida N (2008) Oscillation theory of partial differential equations. World Scientific Publishing, Singapore

\section{Submit your manuscript to a SpringerOpen ${ }^{\circ}$ journal and benefit from:}

- Convenient online submission

- Rigorous peer review

- Immediate publication on acceptance

- Open access: articles freely available online

- High visibility within the field

- Retaining the copyright to your article

Submit your next manuscript at $>$ springeropen.com 